\title{
Selecting candidates for a lower limb stimulator implant programme: A patient-centred method
}

\author{
DN Rushton ${ }^{4}$, FMD Barr ${ }^{2}$, N de N Donaldson ${ }^{1}$, VJ Harper ${ }^{3}$, TA Perkins ${ }^{1}$, PN Taylor ${ }^{3}$ and AM Tromans ${ }^{3}$ \\ ${ }^{1}$ University College, London, Department of Medical Physics \& Bioengineering; ${ }^{2}$ FES Research Unit, Royal National \\ Orthopaedic Hospital, Stanmore; ${ }^{3}$ Spinal Injuries Unit, Salisbury General Hospital; ${ }^{4}$ Department of Rehabilitation, \\ King's College Hospital, London
}

\begin{abstract}
Objective: To develop an effective selection procedure for lower limb functional neurostimulation (LLFNS) for standing in paraplegia.

Design: The selection procedure and exclusion criteria were based on the previous experience of two clinical centres with experience of LLFNS.

Setting: Two Regional Spinal Injuries units in southern England.

Subjects: 254 fully rehabilitated paraplegics living in the community.

Intervention: Patients were invited to participate in the programme, and if suitable to subject themselves to a rigorous staged selection procedure from which they could withdraw at any time.

Outcome measure: Functionally successful home standing using closed-loop surface electrical stimulation.

Results: $57 / 254$ patients were suitable on paper and were accessible. 19 of these $(\mathrm{CI}=10-28)$ were interested in the project and attended one of the spinal centres for details. Twelve $(\mathrm{CI}=5-19)$ of these fulfilled the selection criteria and started on the training programme; and 10 of them completed the muscle training programme successfully. Seven patients $(\mathrm{CI}=2-12)$ achieved closed-loop standing in the laboratory and four patients $(\mathrm{CI}=1-8)$ did so at home.
\end{abstract}

Keywords: paraplegia; electric stimulation; spinal nerve roots

\section{Introduction}

Functional neurostimulation (FNS, or FES) devices are designed to alleviate specific disabilities and improve particular functions. Several requirements must be fulfilled in order to do this. Firstly, a device must work reliably and appropriately, in an engineering sense. Secondly, it must not provoke unacceptable sideeffects. Thirdly, the patient must be neurologically suitable, with the necessary intact peripheral neuromuscular apparatus. Fourthly, the patient must be intellectually and emotionally capable and appropriately motivated to use the device. Fifthly, the patient's lifestyle and commitments, and those of carers and friends, should be such as to enable them to continue to use the device appropriately.

These considerations of course apply to many other aids and treatments for disabled people. The difficulty of ensuring that all the criteria are met, is illustrated by the high proportion of prescribed aids which are either returned as unsuitable, or languish unused in a cupboard after an initial trial. However,

Correspondence: Professor DN Rushton, Frank Cooksey Rehabilitation Unit, King's Healthcare (Dulwich Hospital), East Dulwich Grove, London SE22 8PT there is an additional barrier in the case of FNS devices, which is that they are often surgically implanted. Implantation enables far greater specificity of stimulation and convenience of use; and for many applications, implanted devices are the only effective type. This has several practical consequences. Firstly, it is not possible to 'try out' the device in a realistic way, before making a decision as to whether to proceed with the treatment. Secondly, the emotional 'investment' of the patient and advisers in the success of the device is much larger when a surgical procedure is involved, than when it is not. Thirdly, the commercial cost of implantable devices is usually high, both because of the manufacturing standards required, and the need for manufacturers to be covered for product liability. Fourthly, there is the knowledge that further surgery may be required particularly if the device needs repairing or upgrading in the future.

For these reasons, it is generally accepted that a thorough physical and electrophysiological assessment of potential candidates is essential. An independent psychological assessment looking at patients' motivation and the reality of their aims, has been standard for novel devices, since the beginnings of neural prostheses. But is this enough? We think not. We 
think that the needs of the individual must be taken into account in a much more detailed way. For devices like limb controllers, the selection process must move towards being patient-centred. The patient must be empowered to reach an informed decision as to whether to enter an FES programme, and when. Some patients' interests are likely to be best served by waiting until the device is more developed. Others have reason to wish to be involved at the exciting early stages of a new development, when the outcome is relatively unknown. They must make this choice from a position of self-knowledge and, so far as possible, of technical understanding.

The extent to which the wishes and interests of physically-suitable individuals vary, does depend to some extent on the nature of the device, and the function which it seeks to restore. For example, among the paraplegic population, a high proportion are neurologically suitable for a bladder controller, ${ }^{1}$ and a fairly high proportion of these are dissatisfied with their existing method of bladder management. Almost every paraplegic would like to be continent, free from infection, and able to void on demand. The process of patient selection for implanted bladder controllers is therefore not very difficult. If the protocols ${ }^{2}$ are followed the success rate is very high, and patients continue to use their devices indefinitely. ${ }^{3}$ Normal surgical advice and consent procedures are sufficient.

The same does not seem to apply to limb controllers. For the upper limb controller, which is designed to allow grasp and key-grip functions in the tetraplegic arm, one North American series ${ }^{4}$ showed that $15 / 120$ tetraplegics were assessed as being suitable for the device $(12.5 \%)$, but only four eventually agreed to enter into the research protocol $(3.3 \%)$. There were many physical contraindications, and the training and testing protocol was very time-consuming. Similarly, for lower limb FNS, a survey of patient records ${ }^{5}$ has suggested that only $5-12 \%$ of patients with a thoracic paraplegia may be physically suitable for a stimulator device. The proportion who are physically suitable, willing and able to enter a programme is likely to be even smaller.

Selection procedures for lower limb surface-stimulation FNS programmes have been described before. ${ }^{6}$ The selection process is usually a medical- and therapy-based procedure in which neurological, orthopaedic, cardiovascular, skeletal and ADL indices are tested, and then patients enter an FNS exercise programme. When muscle strength is adequate, patients stand using open-loop and then closed-loop control of standing. Patients largely self-select during this process, for example if they do not have sufficient time or commitment to continue training. The criteria are applied objectively, so far as possible, but in devising a protocol for patient selection for the lumbar anterior root stimulation project, we did not find it practicable to apply the criteria while blind as to the identity of the subject.

\section{Lumbar anterior root stimulator project}

In late 1992 we undertook a project to develop a clinical system for lower limb implant FNS using a multiplexor implanted stimulator ${ }^{7}$ linked to intradural electrodes on the 12 motor roots L2-S2 inclusive, left and right. We foresaw several physiological and surgical advantages to a system of this sort, in comparison with the more usual arrangement whereby stimulating electrodes are placed on the motor nerves in the lower limbs. ${ }^{8}$ However, we were of the view that patient selection would be of crucial importance in determining the success or failure of the project. We therefore developed a patient selection and training protocol, which was based on existing protocols used for surface FNS programmes in the two participating Spinal Injuries Units. We modified them to take account of the fact that this was an implant programme, and combined them so as to apply the same procedure to patients from the two centres.

\section{Selection procedure}

\section{Recruitment}

Both of the Spinal Injuries Units participating in this study have long-standing programmes of limb FES,, 10 and therefore have a pool of patients who are known to be interested. However, we felt that the selection process for this project should initially cast its net as widely as possible. We therefore assessed the patient data banks for both centres, so as to identify all complete traumatic thoracic paraplegics admitted during the preceding 10 years (Salisbury: January 1983-April 1993; Stanmore: January 1982-April 1993). Local Ethical Committee approval was obtained for the study design and information and consent procedures at the involved clinical centres.

We wrote to those patients who appeared suitable on paper, outlining the project and inviting them to contact us if they were interested in taking part, so that they could discuss the project further. An article describing the project and inviting expressions of interest was also published in the Spinal Injuries Association Newsletter. ${ }^{11}$

Respondents expressing an interest were sent a more detailed information sheet, and invited to discuss the project further by telephone or in person. We sent one further letter to the non-respondents.

\section{Selection}

We aimed to design our process so that it selected those patients who were neurologically suitable, had no medical, surgical, psychological or orthopaedic contraindications, were keen to take part, and whose lifestyles would enable them to take full advantage of the programme. We therefore established a sequential selection procedure intended to achieve this. The stages may be summarised as: 
(1) Initial clinical assessment to ensure that the physical criteria were fulfilled. These criteria are detailed below.

(2) The patients completed a 12-week exercise programme using surface stimulation. They had to show a sufficient increase in muscle strength and endurance to enable FES standing.

(3) The patients stood in the laboratory, first with an 'open-loop' system of stimulation control, and then with a 'closed-loop' system. They had to demonstrate safe standing with a satisfactory posture.

(4) The patient used the closed-loop surface stimulation system at home, demonstrating independent safe standing with a satisfactory posture.

Patients had to satisfy the goals of each stage before progressing to the next.

\section{Clinical tests}

Physical inclusion/exclusion criteria The requirements were as follows: (1) Static complete spinal cord lesion between $\mathrm{T} 3-\mathrm{T} 12$ of at least 2 years duration, resulting in a complete upper motor neurone paralysis with a prognosis of no neurological recovery; (2) Age 16-55 years; (3) No medical complications. In particular, patients with cardiovascular or respiratory conditions, or autonomic dysreflexia, were excluded; (4) No lower limb or spinal contractures to prevent upright stance; (5) Suitable mental capacity to enable participation in the programme; (6) No excessive spasticity; (7) No known syringomyelia; (8) No internal spinal fixation instrumentation in the region of the electrode implantation site; (9) Living within 50 miles of one of the participating clinical units.

Measurements We measured and recorded several clinical variables. These included:

Motor and Sensory Neurology - Full body charting of voluntary muscle strength, joint proprioception, and sensation to light touch, deep pressure and temperature.

Joint Range of Movement (ROM) - The passive range is recorded for hips, knees and ankles, to ensure that full extension of hips and knees, and plantargrade positioning of the feet were obtainable. The observations were repeated, to ensure that the ROM did not decrease during the programme.

Spasticity - Three scales were tried: a marked visual analogue scale; an unmarked visual analogue scale; and a $0-5$ rating based on the Ashworth scale. ${ }^{12}$ The latter was found to be the most reliable. Patterns of spastic muscle activity were recorded. Objective measurement of resistance to passive movement was performed using the Wartenburg Pendulum Test, ${ }^{13}$ providing a relaxation index.

Muscle Strength - This was recorded as the peak moment generated during isometric surface electri- cally-driven 1-second contractions of the quadriceps mechanism, with the knee held at 90 degrees flexion. The strength of stimulation was increased until moment readings stabilised. The stimulation strength and moment were recorded. Fatigue resistance was examined by repeated stimulation at the predetermined level, with the knee in 15 degrees flexion. The test continued either for 50 contractions, or until the moment dropped to less than $50 \%$ of the initial value.

Muscle Bulk - Thigh circumference was measured at 10 and $20 \mathrm{~cm}$ above the patella, with the knee in full extension. The two points of measurement of gluteal bulk were at the level of the anterior superior iliac spine and the greater trochanter. Two measurements of calf circumference were taken, at 5 and $15 \mathrm{~cm}$ below the tibial tuberosity. A linear array ultrasound scanner and stand-off gel were used to measure the total depth from skin surface to femur, and to differentiate between subcutaneous fat and the two quadriceps muscle components involved (vastus intermedius and rectus femoris).

Bone Density - Bone mineral content of the neck and shaft of femur and the third, fourth and fifth lumbar vertebrae was examined using a Dexascanner (at Stanmore) or a Novo BMC 22A Gadolinium dual photon bone densitometer (at Salisbury).

Blood Flow - Plethysmography was used to examine cardiac output and stroke volume, and blood flow and pulse volume in the lower limbs.

Muscle Fibre Type and Diameter - A needle biopsy was performed on the vastus lateralis of the dominant leg, and examined for fibre typing and diameter.

Psychological Assessments - Subjects' anxiety and depression levels were measured using the Hospital Anxiety and Depression scale (HAD). Locus of control was examined using the Multi-Dimensional Health Locus of Control Scales. ${ }^{14}$

\section{Results}

\section{Selection before recruitment}

As we expected, it turned out that there was a heavy drop-out at each stage. The first stage is before attempting recruitment. One hundred and fifty-three patients with complete traumatic thoracic paraplegia (rehabilitated during the preceding 10 years) were identified at Salisbury and 101 at Stanmore. When their medical records were examined, 152 of these 254 candidates failed to meet our entry criteria, for the reasons shown in Table 1. A further 45 patients were untraceable, had moved abroad, lived too far away, or were known to be unsuitable. From the 57 remaining patients, and some others who responded to a recruitment notice in the SIA News, ${ }^{11} 19$ volunteers visited one or other of the centres. After further assessment and discussion relating to the study protocol, seven of these volunteers withdrew and the remaining 12 
Table 1 Paper exclusion of subjects

\begin{tabular}{lccc}
\hline Criteria & \multicolumn{2}{c}{ Number of Subjects who failed to meet criterior } & Total \\
Stanmore & Salisbury & 9 & 23 \\
Lower motor neurone paralysis & 14 & 2 & 20 \\
Age & 18 & 10 & 37 \\
Medical complications (including death) & 27 & 7 & 30 \\
Contractures & 23 & 4 & 17 \\
Mental state & 13 & 2 & 4 \\
Excess spasticity & 2 & 1 & 8 \\
Syringomyelia & 7 & 0 & 10 \\
Spinal fixation instrumentation in lumbar spine & 10 & 0 & 3 \\
Living out of area & 3 & 35 & 152 \\
Total & 117 & & \\
\hline
\end{tabular}

Two hundred and fifty-four spinal subjects were considered. These criteria were those which could be applied by examining the records, before contacting the patient

Table 2 Twelve subjects who started the training and assessment programme

\begin{tabular}{|c|c|c|c|c|c|}
\hline \multicolumn{2}{|c|}{ Subject } & \multirow{2}{*}{$\begin{array}{l}\operatorname{Sex} \\
\mathrm{M}\end{array}$} & \multirow{2}{*}{$\begin{array}{c}\begin{array}{c}\text { Age } \\
\text { (years) }\end{array} \\
30\end{array}$} & \multirow{2}{*}{$\begin{array}{l}\begin{array}{l}\text { Level of } \\
\text { injury }\end{array} \\
\mathrm{T} 4\end{array}$} & \multirow{2}{*}{$\begin{array}{c}\text { Time since } \\
\text { injury } \\
\text { (years) }\end{array}$} \\
\hline 1 & $\mathrm{~b}$ & & & & \\
\hline 2 & $\mathrm{~d}$ & $\mathrm{~F}$ & 37 & T3 & 11 \\
\hline 3 & $\mathrm{~b}$ & M & 30 & T5 & 10 \\
\hline 4 & $\mathrm{~d}$ & M & 42 & $\mathrm{~T} 10$ & 4 \\
\hline 5 & $\mathrm{~d}$ & M & 31 & T3L/T4R & 3 \\
\hline 6 & e & $\mathrm{F}$ & 33 & T9 & 3 \\
\hline 7 & $\mathrm{a}$ & M & 27 & T8 & 2 \\
\hline 8 & $\mathrm{~b}$ & M & 37 & $\mathrm{~T} 3$ & 3 \\
\hline 9 & $\mathrm{a}$ & M & 22 & $\mathrm{~T} 4$ & 5 \\
\hline 10 & $\mathrm{c}$ & M & 54 & T8 & 8 \\
\hline 11 & $\mathrm{c}$ & M & 22 & T9 & 4 \\
\hline 12 & $\mathrm{c}$ & M & 40 & $\mathrm{~T} 10$ & 3 \\
\hline
\end{tabular}

Second column (stage reached). a: Failed to complete 12weeks training perod; b: Completed 12 weeks training but did not choose to, or failed to, achieve standing; c: Achieved closed-loop standing in lab only; d: Achieved closed-up loop standing at home; e: Progressed to implantation

started in the programme. Details of these 12 subjects are shown in Table 2.

\section{Selection after recruitment}

The results of the procedures used to select subjects after they had come forward, are summarised in Table 3 . Nine subjects completed the twelve week exercise training period and seven attempted standing using open-loop stimulation. Six were successful, but the remaining subject stood with poor posture and became short of breath after 2 min due to the excessive upper limb work reuqired to keep himself erect. Despite training and additional means of support, posture correction was not achieved and tolerance to standing did not increase, so the subject withdrew from the study. Four of these patients also achieved closed-loop standing, where the stimulation of the quadriceps muscles was controlled by the knee angle during standing; stimulation is switched off during full extension.

Two other subjects completed the exercise period but did not attempt standing with the stimulation. The first experienced a substantial increase in the level of spasticity, such that he was unable to function at his previous level and was forced to retire from the programme. The second subject stimulated for a total of 23 weeks but failed to increase muscle bulk or strength and therefore did not meet the criterion set for standing. Two further subjects withdrew from the study during the exercise training phase. One subject experienced considerable difficulty finding time to stimulate due to other commitments and the other subject attributed a decrease in his sexual function to the stimulation.

\section{Results of tests}

Motor and sensory neurology No change in voluntary muscle power, joint proprioception or sensation to light touch, deep pressure or temperature was observed in any of the subjects.

Joint range of movement No change in joint range of movement in the hips, knees and ankle joints was observed in any of the subjects.

Spasticity Subjective ratings and objective measurements of spasticity and its influence on the ability to perform functional activities were performed. These are shown in Table 4, for the 11 subjects who reached this stage. There was a subjective increase in spasticity in 7/11 (one Ashworth point in each case). On objective testing, three of these subjects also had a bilateral increase in objective spasticity (relaxation index reduced). Three other legs showed a unilateral reduction in relaxation index. Two patients showed a slight bilateral increase in relaxation index, and two other legs showed an increase in relaxation index. Two 
Table 3 Dropout $v$ Stage

\begin{tabular}{|c|c|c|}
\hline Stage in selection procedure & Number & $\%$ Remaining \\
\hline Total paraplegic population identified* & 254 & 100 \\
\hline Unsuitable on paper (see Table 1 ) & 152 & 40 \\
\hline Suitable on paper but untraceable & 45 & 22 \\
\hline Sent letters inviting their participation & 57 & 22 \\
\hline Volunteers & 19 & 7.5 \\
\hline Entered into programme of training & 12 & 4.7 \\
\hline Completed muscle training programme & 10 & 3.9 \\
\hline Achieved open-loop standing & 7 & 2.8 \\
\hline Achieved closed-loop standing at home & 4 & 1.6 \\
\hline
\end{tabular}

* One of those who responded to the SIA newsletter was suitable and is included in this table

Table 4 Spasticity Measurements: Ashworth scale and wartenberg index (absolute changes). Before and after 12week muscle training programme

\begin{tabular}{lccc}
\hline & $\begin{array}{c}\text { Subjective: } \\
\text { Ashworth } \\
\text { Scale }\end{array}$ & $\begin{array}{c}\text { Objective: } \\
\text { R. } \text { Wartenberg index } \\
\text { relaxation } \\
\text { index }\end{array}$ & $\begin{array}{c}\text { L. leg } \\
\text { relaxation } \\
\text { index }\end{array}$ \\
\hline Subject & NC & 0.01 & 0.71 \\
2 & NC & 0.2 & 0.2 \\
3 & NC & 0.04 & -0.02 \\
4 & $2-3$ & 0.04 & -0.56 \\
5 & $3-4$ & 0 & 0 \\
6 & $2-3$ & -0.04 & -0.59 \\
8 & $2-3$ & no data & no data \\
9 & $3-4$ & -0.29 & -0.04 \\
10 & $3-4$ & -0.25 & -0.15 \\
11 & $3-4$ & -0.2 & 0 \\
12 & NC & -0.01 & 0 \\
\hline
\end{tabular}

For Ashworth Scale, $\mathrm{NC}=$ no change. (Done in $11 / 12$ patients)

patients showed a relaxation index reduction in one leg, and an increase in the other.

Muscle strength The change, during the training period, in peak torque and fatigue index for the quadriceps mechanism in the same 11 subjects is shown in Table 5. Eight subjects showed an increase in peak torque in the left leg, and 10 in the right. Endurance was measured by repeated stimulation at a predetermined level, with the knee held at 15 degrees of flexion. Endurance increased bilaterally in four patients, and unilaterally in two more. Endurance decreased bilaterally in one patient; in the remaining three patients it was unchanged. The one patient who showed little increase in either strength or endurance (strength actually decreased in one leg) withdrew from the programme for this reason.

Muscle bulk Changes in thigh and calf circumference (two levels for each, in each leg) and gluteal bulk are shown in Table 6 as percentage change, for the same nine patients. Of these 90 percentages, 71 show an increase in
Table 5 Changes in muscle strength and endurance changes in newton-metres (quadriceps) (Done in 11/12 patients)

\begin{tabular}{lcccc}
\hline & \multicolumn{2}{c}{$\begin{array}{c}\text { Maximal moment } \\
(\text { Nm) }\end{array}$} & \multicolumn{2}{c}{$\begin{array}{c}\text { Contractions to } \\
\text { fatigue (change, sec) }\end{array}$} \\
Subject & Right leg & Left leg & Right leg & Left leg \\
\hline 1 & 66 & 64 & 35 & -6 \\
2 & 7.9 & 4.5 & 0 & 0 \\
3 & 21 & -17.2 & 0 & 0 \\
4 & 10.1 & 3.2 & -24 & 5 \\
5 & 7.9 & 6.5 & 0 & 0 \\
6 & 16.7 & 12 & 17 & 19 \\
8 & 8.3 & 2.9 & 19 & 17 \\
9 & 8.5 & -0.6 & 5 & 7 \\
10 & 2.8 & -5.2 & 29 & 25 \\
11 & 40 & 36 & -13 & -11 \\
12 & 16.1 & 7.4 & 0 & 0 \\
\hline
\end{tabular}

bulk, 11 a decrease, while eight show no change. This test was not a selection criterion. Linear array ultrasound scan measurements demonstrated increased quadriceps thickness in five of the six subjects measured (this test was only done at Salisbury).

Bone density Data are incomplete, owing to difficulties with the dexascanner source. For the femoral neck, no age-matched control data were available for European populations. Figures were therefore compared with the closest available data (North American females). Of the seven patients for whom data are available, all except one were within or above two standard deviations (SD) of the normal mean. One male patient was just below; he later withdrew for other reasons. Initial bone density in lumbar spine was within $2 \mathrm{SD}$ of normal for age and sex in all seven. However, one male subject showed a decrease in density during the training period, and subsequently withdrew from the project for other reasons. For femoral shafts there were no age-matched control data.

Blood flow Data are available for five patients (Table $8)$. In the lower limbs (10 limbs), stroke volume increased during the training period in five, was unchanged in four, and fell in one. Blood flow 
Table 6 Changes in muscle bulk (Tape measured circumference at stated sites-done in 10/12 patients)

\begin{tabular}{|c|c|c|c|c|c|c|c|c|c|c|}
\hline $\begin{array}{l}\text { Level of } \\
\text { measurement }\end{array}$ & $A S I S$ & $G T$ & $\begin{array}{l}L Q \\
10 \mathrm{~cm}\end{array}$ & $\begin{array}{c}R Q \\
10 \mathrm{~cm}\end{array}$ & $\begin{array}{c}L Q \\
20 \mathrm{~cm}\end{array}$ & $\begin{array}{c}R Q \\
20 \mathrm{~cm}\end{array}$ & $\begin{array}{l}L C \\
5 \mathrm{~cm}\end{array}$ & $\begin{array}{l}R C \\
5 \mathrm{~cm}\end{array}$ & $\begin{array}{c}L C \\
15 \mathrm{~cm}\end{array}$ & $\begin{array}{c}R C \\
15 \mathrm{~cm}\end{array}$ \\
\hline $\begin{array}{l}\text { Mean \% } \\
\text { increase }\end{array}$ & 3.2 & 2.4 & 3.4 & 4.1 & 2.9 & 2.3 & 2.4 & 3.3 & 3.8 & 4.0 \\
\hline $\begin{array}{l}\text { Increase } \\
\text { (number of } \\
\text { subjects) }\end{array}$ & 6 & 6 & 8 & 9 & 9 & 7 & 9 & 8 & 8 & 7 \\
\hline $\begin{array}{l}\text { ISQ (number } \\
\text { of subjects) }\end{array}$ & 3 & 2 & 1 & 0 & 0 & 1 & 0 & 2 & 0 & 1 \\
\hline $\begin{array}{l}\text { Decrease } \\
\text { (number of } \\
\text { subjects) }\end{array}$ & 1 & 2 & 1 & 1 & 1 & 2 & 1 & 0 & 2 & 2 \\
\hline
\end{tabular}

Key: $\mathrm{ASIS}=$ anterior superior iliac spine; $\mathrm{GT}=$ greater trochanter; $\mathrm{LQ}=$ left quadriceps; $\mathrm{RQ}=$ right quadriceps; $\mathrm{LC}=$ left calf; $\mathrm{RC}=$ right calf; $\mathrm{ISQ}=$ no change

Table 7 Changes in cardiac output and stroke volume, and lower limb blood flow and stroke volume (Done in 8/12 patients, $16 / 24$ legs)

\begin{tabular}{lcccc}
\hline $\begin{array}{l}\text { Site and number } \\
\text { of measurements }\end{array}$ & $\begin{array}{c}\text { Cardiac stroke } \\
\text { volume }(/ 8)\end{array}$ & $\begin{array}{c}\text { Cardiac output } \\
(/ 8)\end{array}$ & $\begin{array}{c}\text { Lower limb blood flow }(R \\
\text { and L legs) }(/ 16)\end{array}$ & $\begin{array}{c}\text { Lower limb stroke } \\
\text { volume }(/ 10)\end{array}$ \\
\hline Increase & 3 & 7 & 10 & 5 \\
ISQ & 4 & 0 & 4 & 4 \\
Decrease & 1 & 1 & 2 & 1 \\
\hline
\end{tabular}

increased in nine limbs, and decreased in one. This test was not a selection criterion.

Muscle fibre type and diameter Data analysis is incomplete at present. This test was not a selection criterion.

Psychological assessments All nine subjects scored within the normal range for anxiety and depression at the beginning and end of training. However, there were changes. Six subjects showed a decrease in their anxiety score during the training period, and four showed a decrease in the depression score. There was no significant change in the scores for locus of control. No patient was withdrawn as a result of this test.

\section{Discussion}

The pattern of drop-out, with a marked attrition at each stage in the selection process, was an interesting finding. The initial weeding-out on clinical grounds was in line with our previous experience and with the literature. However an important finding was the significant number of volunteers who dropped out during or after training, or who proved to be unsuitable at this stage. Of the 102 patients who were suitable on paper, 57 were accessible and 19 volunteered. Assuming our paraplegic population is representative, this means that there is a $95 \%$ probability that between 10 and 28 volunteers would be obtained from a suitable population of 102 (that is,
Table 8 Changes in mood and locus of control (Done in 9/2 patients)

\begin{tabular}{lccccc}
\hline & \multicolumn{5}{c}{ Number of subjects } \\
Scale & Anxiety & Depression & IHLC & PHLC & CHLC \\
\hline Increase & 1 & 0 & 2 & 2 & 2 \\
ISQ & 1 & 5 & 1 & 0 & 1 \\
Decrease & 7 & 4 & 6 & 7 & 6 \\
\hline
\end{tabular}

Key: $\quad$ IHLC = Internal Health Locus of Control; PHLC $=$ Powerful Others Health Locus of Control; $\mathrm{CHLC}=$ Chance Health Locus of Control

the $95 \%$ confidence interval $\mathrm{CI}$ is $10-28)$. Of the 19 , 12 started training $(\mathrm{CI}=5-19)$; seven achieved laboratory closed-loop standing $(\mathrm{CI}=2-12)$ and four achieved home closed-loop standing $(\mathrm{CI}=1-8)$. A search of this size is therefore necessary in order to be quite confident that suitable patients will be found.

The results emphasise the importance of the initial selection procedure; muscle training is the first stage at which a considerable investment of time is made by both patient and investigator. It is essential to do as much selection as possible before this stage. Because of the degree of user participation required in limb FNS, the lifestyle, potential commitment, occupation and free-time of the user are all of crucial importance.

Because the number of suitable subjects is limited, after the selection process, it follows that many will live far away from the centre. For a project like this, 
which includes many testing visits, long home training periods, and equipment to maintain, the logistics of dealing with far-flung patients become significant. In practice, this limits the number of available subjects to those living with a 50-mile radius of one or other of the Spinal Injuries Units. In order to extend the programme to a greater number of suitable patients, while maintaining the selection and training criteria, it would be necessary to involve more than two Spinal Injuries centres. Four of the 12 volunteers who actually entered the training programme (33\%) succeeded in reaching all the goals required in order to proceed to implantation. Although these represented only $1.6 \%$ of the original paraplegic population identified, they comprised $21 \%$ of those who put themselves forward for the programme.

\section{Acknowledgements}

This work was supported by the Medical Research Council, and the charities ASPIRE and INSPIRE

\section{References}

1 Brindley GS, Polkey CE, Rushton DN, Cardozo L. Sacral anterior root stimulators for bladder control in paraplegia: the first 50 cases. J Neurol Neurosurg Psychiat 1986; 49: 1104-1114.

2 Brindley GS. The Sacral Anterior Root Stimulator: Notes for Surgeons and Physicians. Obtainable from Finetech Ltd, 13 Tewin Court, Welwyn Garden City, Herts, UK.

3 Brindley GS, Rushton DN. Long-term follow-up of patients with sacral anterior root stimulator implants. Paraplegia 1990; 28: $469-475$.
4 Gorman PH et al. Patient selection for an upper extremity functional electrical stimulation neuroprosthesis. In: Proceedings, 25th Annual Neural Prosthesis Workshop: NIH, Bethesda, Md, 1994.

5 Jaeger RJ, Yarkony GM, Roth EJ, Lovell L. Estimating the user population of a simple electrical stimulation system for standing. Paraplegia 1990; 28: 505-511.

6 Schlecht $\mathrm{M}$ et al. Selection and training of patients for FES. In: van Alste J (ed) Restoration of Walking aided by Functional Electrical Stimulation. Edizione Fondazione Pro Juventute, Milan, 1987: pp 75-78.

7 Donaldson N de N. A new multiplexed stimulator for FNS. In: Popovic DB (ed) Advances in External Control of Human Extremities, $X$. Belgrade, Nauka, 1990: pp 345-358.

8 Rushton DN. Choice of nerves or roots for multichannel leg controller implants. In: Popovic D (ed) Advances in External Control of Human Extremities X. Belgrade: Nauka, 1990: pp 99108.

9 Swain ID. Conditioning of skeletal muscle by long term functional electrical stimulation-implications for the development of practical systems. In: Illis L (ed) Spinal Cord Dysfunction, Vol III: Functional Stimulation. Oxford: OUP, 1992: pp 175-205.

10 Barr FMD, Bayley JIL, Middleton FRI, Moffat B. Functional electrical stimulation: practical experience in the clinical setting. In: Popovic DB (ed) Advances in External Control of Human Extremities, IX. Belgrade: Yugoslav Committee for Electronics and Automation, 1987: pp 181-191.

11 Spinal Injuries Association Newsletter No 71 (November 1993).

12 Ashworth B. Preliminary trial of carisoprodol in multiple sclerosis. Practitioner 1964; 192: 540 - 542.

13 Bajd T, Bowman RG. Testing and modelling of spasticity. $J$ Biomed Eng 1982; 4: 90 -96.

14 Wallston KA, Wallston BS. Health Education Monographs 1978; 6: $160-170$. 